$$
\text { CONF-9606226--7 }
$$

\title{
NONDIMENSIONAL TRANSPORT EXPERIMENTS ON DIII-D AND PROJECTIONS TO AN IGNITION TOKAMAK
}

\author{
by \\ C.C. PETTY, T.C. LUCE, B. BALET, \\ J.P. CHRISTIANSEN, and J.G. CORDEY
}

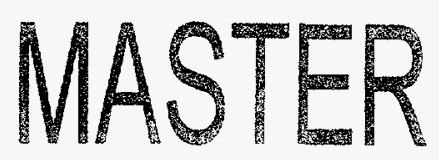

DISTRIBUTION OF THES DOCMENT IS UMUATED

JULY 1996 


\section{DISCLAIMER}

This report was prepared as an account of work sponsored by an agency of the United States Government. Neither the United States Government nor any agency thereof, nor any of their employees, makes any warranty, express or implied, or assumes any legal liability or responsibility for the accuracy, completeness, or usefulness of any information, apparatus, product, or process disclosed, or represents that its use would not infringe privately owned rights. Reference herein to any specific commercial product, process, or service by trade name, trademark, manufacturer, or otherwise does not necessarily constitute or imply its endorsement, recommendation, or favoring by the United States Government or any agency thereof. The views and opinions of authors expressed herein do not necessarily state or reflect those of the United States Government or any agency thereof. 


\section{DISCLAMMER}

Portions of this document may be illegible in electronic image products. Images are produced from the best available original document. 
GA-A22385

\title{
NONDIMENSIONAL TRANSPORT EXPERIMENTS ON DIII-D AND PROJECTIONS TO AN IGNITION TOKAMAK
}

\author{
by \\ C.C. PETTY, T.C. LUCE, B. BALET, ${ }^{*}$ \\ J.P. CHRISTIANSEN, ${ }^{\star}$ and J.G. CORDEY*
}

This is a preprint of a paper to be presented at the Twenty-Third European Conference on Controlled Fusion and Plasma Physics, June 24-28, 1996, Kiev, Ukraine, and to be published in The Proceedings.

*JET Joint Undertaking, United Kingdom.

\author{
Work supported by \\ the U.S. Department of Energy \\ under Contract No. DE-AC03-89ER51114
}




\title{
NONDIMENSIONAL TRANSPORT EXPERIMENTS ON DIII-D AND PROJECTIONS TO AN IGNITION TOKAMAK*
}

\author{
C.C. Petty, T.C. Luce, B. Balet, ${ }^{\dagger}$ J.P. Christiansen,${ }^{\dagger}$ J.G. Cordey ${ }^{\dagger}$ \\ General Atomics, P.O. Box 85608, San Diego, California 92186-9784 U.S.A.
}

The concept of nondimensional scaling of transport makes it possible to determine the required size for an ignition device based upon data from a single machine and illuminates the underlying physics of anomalous transport. The scaling of cross-field heat transport with the relative gyroradius $\rho_{*}$, the gyroradius normalized to the plasma minor radius, is of particular interest since $\rho_{*}$ is the only nondimensional parameter which will vary significantly between present day machines and an ignition device. These nondimensional scaling experiments are based upon theoretical considerations 1,2 which indicate that the thermal heat diffusivity can be written in the form

$$
\chi=\chi_{B} \rho_{*}^{x_{\rho}} F\left(\beta, v_{*}, q, R / a, \kappa, T_{e} / T_{i}, \ldots\right),
$$

where $\chi_{B}=c T / e B$. As explained elsewhere, ${ }^{3} x_{\rho}=1$ is called gyro-Bohm scaling, $x_{\rho}=0$ is Bohm scaling, $x_{\rho}=$ $-1 / 2$ is Goldston scaling, and $x_{\rho}=-1$ is stochastic scaling. The DIII-D results reported in this paper cover three important aspects of nondimensional scaling experiments: the testing the underlying assumption of the nondimensional scaling approach, the determination of the $\rho_{*}$ scaling of heat transport for various plasma regimes, and the extrapolation of the energy confinement time to future ignition devices.

\section{DIMENSIONALLY IDENTICAL H-MODE DISCHARGES}

A basic assumption of the nondimensional scaling approach is that transport is dependent only upon local quantities. Therefore if two plasmas with different $B$ and $a$ are constructed which have the same values for all the nondimensional parameters $\left(\rho_{*}, \beta, v_{*}, q, R / a, \kappa, e t c\right.$ ), then it can be shown from Eq. (1) that the thermal energy confinement times normalized to the cyclotron frequencies (i.e., $B \tau_{t h}$ ) should also be the same for these two plasmas. Plasmas which have the same values for all the nondimensional parameters are referred to as dimensionally identical discharges.

Recently experiments on DIII-D and JET have compared the confinement of dimensionally identical discharges for the first time using plasmas with substantially different $B$ and $a$. The JET discharge to be matched on DIII-D was selected from a previously published $\rho_{*}$ scaling experiment (shot 35171 in Ref. 4). It is a stationary ELMing $\mathrm{H}$-mode discharge with $\beta_{\mathrm{N}}=1.6$ and the same plasma shape as expected for ITER. Neutral beam injection (NBI) heating was utilized on both JET and DIII-D for these experiments.

The normalized plasma parameters for the DIII-D and JET H-mode discharges were well matched, as shown in Table I. The aspect ratio, elongation, and safety factor were the same to about $1 \%$. In order to keep $\rho_{*}, \beta_{t h}$, and $v_{*}$ fixed for these two discharges with different $B$ and $a$, the quantities $B a^{5 / 4}, W_{t h} / a^{1 / 2}$, and $\bar{n} a^{2}$ were also held constant by adjusting the toroidal field, the injected power, and the density. The ELM frequencies $(\mathrm{ELM})$ normalized to the thermal confinement times were similar although not identical for these two plasmas. Table I shows that the thermal confinement time (with the NBI power corrected for shinethrough losses) normalized to the cyclotron frequency for the DIII-D and JET dimensionally identical $\mathrm{H}$-mode discharges agreed to within $3 \%$, which is well inside the experimental error bars. Thus plasmas which have identical values for the local dimensionless parameters have been shown to have identical

\footnotetext{
*Work supported by the U.S. Department of Energy under Contract No. DE-AC03-89ER51114.

$\dagger$ Permanent address: JET Joint Undertaking, United Kingdom.
} 
normalized confinement, which demonstrates that nondimensional scaling is a valid approach to understanding transport processes.

\section{$\rho$ * SCALING OF HIGH $q \mathrm{H}-\mathrm{MODES}$}

Previous experiments on DIII-D which measured the $\rho_{*}$ scaling of ions and electrons found that the ion $\rho_{*}$ scaling varied according to the plasma regime. In $\mathrm{H}$-mode plasmas with low safety factor $\left(q_{95}=3.8\right)$, the ion scaling was gyroBohm-like, ${ }^{5}$ whereas in L-mode plasmas with the same safety factor, Bohm-like ion scaling was observed. ${ }^{6}$ The $\rho_{*}$ scaling for electrons was always found to be gyro-Bohm-like. Comparing the transport results for these $\mathrm{H}$-mode and $\mathrm{L}$-mode plasmas indicated a correlation between the ion $\rho_{*}$ scaling and the density scale length, ${ }^{6}$ i.e., as the density profile became more peaked the ion $\rho_{*}$ scaling varied from gyroBohm-like to Bohm-like.

Recent experiments on DIII-D have looked for a similar correlation between the ion $\rho_{*}$ scaling and the safety factor scale length. This was motivated by the observation that the ion $\rho_{*}$ scaling changed from Bohmlike to Goldston-like between low- $q$ L-mode ${ }^{6}$ and high- $q$ L-mode ${ }^{3}$ experiments. For this experiment, the $\rho_{*}$ scaling of transport was measured on DIII-D in high safety factor $\left(q_{95}=7\right)$ ELMing H-mode discharges with flat density profiles (minimizing the influence of the density scale length) for comparison with the $\rho_{*}$ scaling of the ITER-relevant low- $q \mathrm{H}$-mode discharges previously studied..$^{5}$ The aspect ratio, elongation, $T_{e} / T_{i}$, and $\beta_{\mathrm{N}}$ were nearly the same for these two pairs of dimensionally similar discharges. The radial profiles of four important nondimensional parameters for the high- $q \mathrm{H}$-mode discharges are shown in Fig. 1, demonstrating that the dimensionless quantities were held fixed as $\rho_{*}$ was varied. The ratio of the ion thermal diffusivities for this $\rho_{*}$ scan are shown in Fig. 2. The ion diffusivity had gyro-Bohm-like scaling in the inner half of the plasma, changing to Bohm-like scaling in the outer half of the plasma; this should be compared to gyroBohm-like scaling for the low- $q \mathrm{H}$-mode. The safety factor scale length for the high- $q \rho_{*}$ scan differs from the low- $q \rho_{*}$ scan mainly in the outer half of the plasma since sawtooth activity enforces the same $q$ profile in the inner region. Therefore, the change in the ion $\rho_{*}$ scaling from gyro-Bohm-like to Bohm-like correlates with a shortening of the safety factor scale length. This indicates that a peaked current profile as well as a peaked density profile can lead to worse than gyro-Bohm scaling for the ions.

\section{PROJECTIONS TO AN IGNITION TOKAMAK}

The confinement properties of future magnetic fusion devices can be extrapolated from existing experiments using the scaling of heat transport with nondimensional parameters such as $\rho_{*}$. The $\rho_{*}$ scaling of dimensionally similar discharges have been compared between DIII-D and JET for H-mode plasmas in order to test the extrapolation of heat transport to an ignition device. Using Eq. (1), the loss (transported) power for plasmas with different $\rho_{*}$ but the same $\beta, v_{*}$, etc., can be shown to scale like $P_{\text {loss }} \propto \rho_{*}^{-5 / 2+x_{\rho}} a-3 / 4$. The loss power projected along a dimensionally similar path for the ITER-relevant low- $q \mathrm{H}$-mode plasmas on DIII-D which had gyro-Bohm-like scaling 5 is shown in Fig. 3 . The favorable gyro-Bohm-like scaling extrapolates to a very low loss power (high confinement) for ITER.

As discussed in Ref. 6, however, this extrapolation from DIII-D to smaller $\rho_{*}$ on ITER is complicated by the requirement that the loss power from core transport remain above the $\mathrm{H}$-mode threshold power along the dimensionally similar path (in order for the plasma to remain in $\mathrm{H}$-mode). Since the $\mathrm{H}$-mode threshold power scales like $P_{t h r} \propto \rho_{*}^{-3} a^{-3 / 4,7}$ which is Goldston-like, the increase in the H-mode threshold power is 
more rapid than the increase in loss power as $\rho_{*} \rightarrow$ 0 . Data from JET plasmas ${ }^{4}$ (see Fig. 3) shows that when $P_{\text {loss }}$ drops to the level of $P_{t h r}$ as $\rho_{*}$ is decreased, the dimensionally similar path changes from gyro-Bohm-like scaling, which follows core transport, to Goldston-like scaling, which follows the $\mathrm{H}$-mode threshold.

Choosing a scaling path at high beta (and low collisionality), along which the loss power remains above the $\mathrm{H}$-mode threshold to the point of ignition, allows one to take full advantage of the gyro-Bohmlike scaling of heat transport in $\mathrm{H}$-mode plasmas in the design of an ignition tokamak. An example of this is given in Fig. 3, where an ignition point along a dimensionally similar path at higher $\beta$ than the ITER-relevant DIII-D and JET discharges is shown. If beta is increased by raising the temperature at fixed density, then the $\mathrm{H}$-mode threshold powers are the same for the high $\beta$ and low $\beta$ paths. Since ignition takes place for $P_{\text {loss }}$ slightly above $P_{t h r}$ along the high $\beta$ path, there is enough power flow through the edge to sustain the $\mathrm{H}$-mode condition. The parameters for this high $\beta$ "compact-gB" ignition point are given in Table II; the reduced minor radius compared to ITER means a smaller extrapolation is needed from existing machines like DIII-D and JET and the density is lower relative to the Greenwald limit. The high $H$ factor is a natural consequence of the favorable gyro-Bohm-like scaling of $\mathrm{H}$-mode confinement.

The high value of $\beta_{N}$ for the compact-gB scenario is a result of the low elongation and low triangularity of the assumed ITER shape. By utilizing a more highly shaped plasma, the normalized beta can be reduced without sacrificing performance. The parameters for such a " $D$ " shaped $\mathrm{H}$-mode plasma are given in Table II, where the ignition point was determined by the same procedure as for the compact-gB point shown in Fig. 3. It is noteworthy that the operating point for the compact-D scenario does not exceed either the Troyon beta limit or the Greenwald density limit.

\section{CONCLUSIONS}

Comparison of dimensionally identical $\mathrm{H}$-mode discharges on DIII-D and JET found that the thermal confinement times normalized to the cyclotron frequencies were in good agreement,
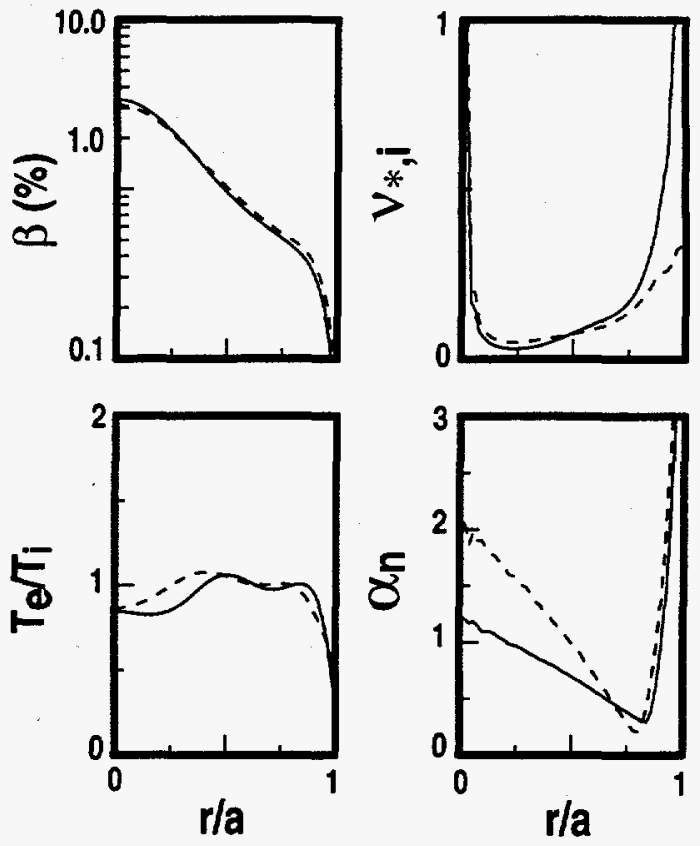

Fig. 1. Nondimensional parameters for the high$q \mathrm{H}$-mode $\rho_{*}$ scan. The solid lines are for $\mathrm{B}=$ $2.1 \mathrm{~T}$ and the dashed lines are for $\mathrm{B}=1.05 \mathrm{~T}$. The quantity $\alpha$ is the power to which a parabolic radial dependence must be raised to get the local scale length.

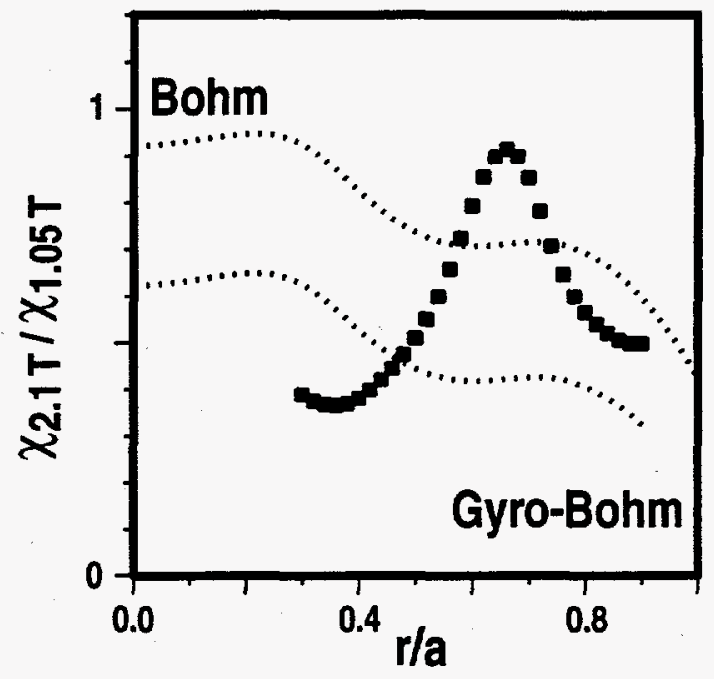

Fig. 2. Ratio of ion thermal diffusivities for the high- $q \mathrm{H}$-mode discharges. The expected ratios for gyro-Bohm-like and Bohm-like scaling are also shown, postulating that $\chi_{\mathrm{gB}} \propto \mathrm{T}_{\mathrm{i}}^{3 / 2} / B^{2}$ and $\chi_{\mathrm{B}} \propto \mathrm{T}_{\mathrm{i}} / B$. 


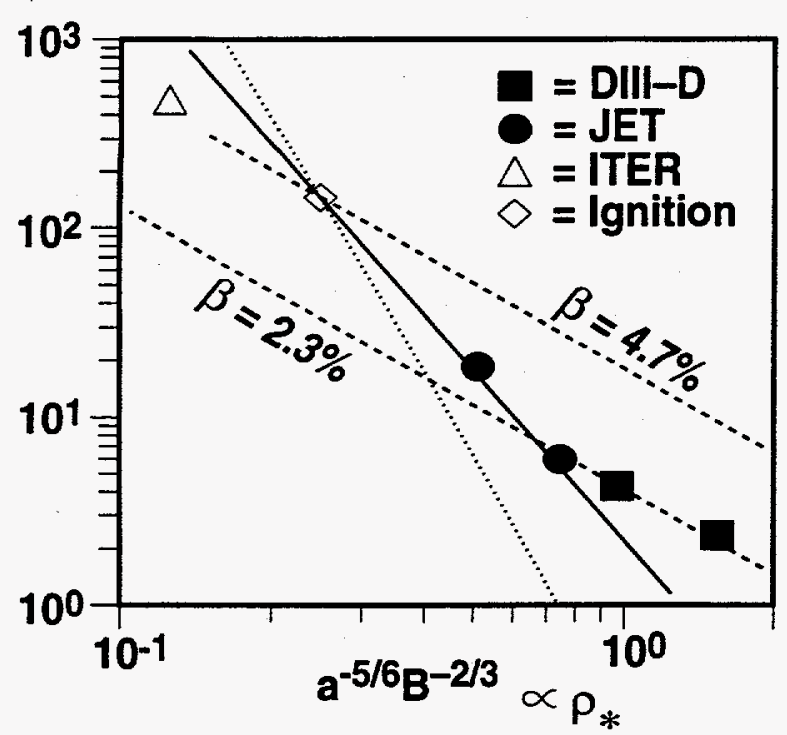

Fig. 3. Dimensionally similar paths for the loss power of DIII-D and JET H-mode discharges, the projected loss power for gyro-Bohm-like scaling at two values of $\beta$ (dashed lines), $H$-mode threshold power (solid line), and the alpha loss power for the high $\beta$ path (dotted line).
Table II: Projected parameters for gyro-Bohm ignition tokamaks with $R / a=2.7, q_{95}=3.8$, and $B_{T}=5.7 \mathrm{~T}$.

\begin{tabular}{lcc}
\hline Parameter & Compact-gB & Compact-D \\
\hline$R(\mathrm{~m})$ & 3.56 & 2.74 \\
$\kappa$ & 1.68 & 2.1 \\
$\delta$ & 0.36 & 0.8 \\
$I(\mathrm{MA})$ & 8.5 & 4.9 \\
$\bar{n}\left(10^{20} \mathrm{~m}^{-3}\right)$ & 1.9 & 2.0 \\
$\bar{n} / n_{\mathrm{GW}}$ & 1.2 & 0.65 \\
$\beta_{\mathrm{N}}$ & 4.7 & 3.3 \\
$\tau(\mathrm{s})$ & 1.4 & 1.2 \\
$\mathrm{H}$ & 3.3 & 3.0 \\
$P_{\text {fus }}(\mathrm{MW})$ & 660 & 490 \\
\hline
\end{tabular}

indicating that transport is a function of only the local quantities and that Eq. (1) is a valid representation of the transport process. Other experiments on DIII-D showed that the $\rho_{*}$ scaling for ions varied from gyro-Bohm-like for plasmas with broad density and current profiles to worse than Bohm-like for peaked density and current profiles. Finally, it was shown that the gyro-Bohm-like scaling of low- $q \mathrm{H}$-mode plasmas can lead to an attractive compact ignition tokamak by choosing a high beta (low collisionality) path along which the loss power remains above the $\mathrm{H}$-mode threshold power to the point of ignition.

${ }^{1}$ Kadomtsev, B.B., Sov. J. Plasma Phys. 1, 295 (1975).

${ }^{2}$ Connor, J.W., and Taylor, J.B., Nucl. Fusion 17, 1047 (1977).

${ }^{3}$ Petty, C.C., et al., Phys. Rev. Lett. 74, 1763 (1995).

${ }^{4}$ Balet, B., et al., in the Proc. of the 22nd Euro. Conf. on Contr. Fusion and Plasma Physics, Bournemouth, (European Physical Society, Petit-Lancy, Switzerland, 1995) Vol. 19C, Part I, p. 9.

5Petty, C.C., et al., Phys. Plasmas 2, 2342 (1995).

${ }^{6}$ T.C. Luce and C.C. Petty, in the Proc. of the 22nd Euro. Conf. on Contr. Fusion and Plasma Physics, Bournemouth, (European Physical Society, Petit-Lancy, Switzerland, 1995) Vol. 19C, Part III, p. 25.

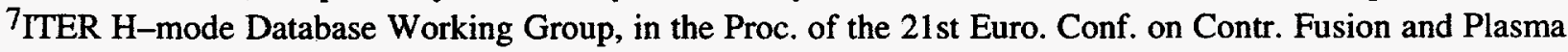
Physics, Montpellier, (European Physical Society, Petit-Lancy, Switzerland, 1994) Vol. 18B, Part I, p. 334. 